\title{
Critical Aortic Stenosis in a Patient with a Abdominal Aort Dissection and Narrowed: Simultaneous Transcatheter Aortic Valve Implantation and Endovascular Aortic Repair
}

\author{
Refik Emre Altekin Md ${ }^{{ }^{*}}$, Alı Yasar Kılınc Md ${ }^{1}$, Gorkem Goldag Md ${ }^{1}$ \\ ${ }^{1}$ Akdeniz University Faculty of Medicıne Department of Cardıology, Turkey \\ *Asisstance Professor, Akdeniz University Hospital Department of Cardiology, Dumlupinar Bouleward, \\ Konyaalti, Antalya, Turkey
}

*Corresponding Author: Refik Emre Altekin Md, Asisstance Professor, Akdeniz University Hospital Department of Cardiology, Dumlupinar Bouleward, Konyaalti, Antalya, Turkey, Email: dremre29 @yahoo.com

\begin{abstract}
A 76-year-old male patient presented with symptoms and signs of heart failure. Echocardiographic evaluation revealed severe aortic stenosis(aortic valve area: $0.7 \mathrm{~cm} 2$ ). EUROSCORE II:15.6\%, STS:11\% for the patient's aortic valve surgery. The patient with high risk for surgery is planned for TAVI. Abdominal level aortic dissection in the abdominal aorta at the preoperative computed tomography was not found to be sufficient for TAVI operation of the true lumenal opening in the region concerned. The dissection area was treated with the endovascular aortic repair and sufficient lumen clearance was obtained and in the same seance TAVI was performed. If the presence of abdominal aortic dissection or aneurysm in the TAVI patients due to aortic stenosis is not noticed, it can lead to serious complications. The Aortic Endovascular Repair(EVAR) and Transcatheter Aortic Valve Implantation(TAVI) procedure, which will be performed in the same seance for the prevention of complications, is an effective and reliable treatment approach.
\end{abstract}

Keywords: Aortic Stenosis, Aortic Dissection, TAVI, EVAR

\section{LEARNING OBJECTIVE}

Patients with advanced age aortic stenosis may be accompanied by aortic dissection or aneurysm. Especially, it is important to examine the tomography in the detection of such pathologies before TAVI treatment. Aortic aneurysm or dissection may lead to lethal complications during TAVI procedure. In such clinical situations, TAVI and EVAR procedures can be performed safely in the same seance.

\section{INTRODUCTION}

Transcatheter aortic valve implantation (TAVI) is currently considered an acceptable alternative for the treatment of patients with severe aortic stenosis and a high perioperative risk or a contraindication for open surgery(1). Even TAVI has been shown to be successful and safe, the cardiovascular assessment of other comorbidities in these patients is critical for ensuring better clinical outcomes(2). Particularly, aorta associated pathologies can lead to fatal complications during TAVI procedure. Tomographic examinations that are performed prior to TAVI treatment can help detecting and treating related pathologies and prevent complications(3).

We report a case of an elderly patient with an abdominal aortic dissection and critical abdominal aortic lumen stenosis as well as critical symptomatic aortic stenosis. The patient subsequently underwent percutaneous endovascular aortic dissection repair(EVAR) immediately followed by a successful TAVI.

\section{CASE RePORT}

An 76-year-old man was initially admitted to hospital with dyspnea, orthopnea, scrotum and ankle swelling. Functional capacity of the patient was NYHA Class 4. He had multiple comorbidities including chronic renal failure(grade 4) due to diabetic nephropathy ,hypertension and type 2 diabetes(IDDM).

Electrocardiography showed sinus rhythm and left branch block. Cardiomegaly, interstitial edema and pleural effusion were observed with 
the telecardiography. A transthoracic echo cardiogram showed critical aortic stenosis (aortic mean gradient of $45 \mathrm{~mm} \mathrm{Hg}$ and valve area of $0.7 \mathrm{~cm} 2$ ) with mild concentric left ventricular hypertrophy and mildly reduced left ventricular systolic (LVEF:\%50) and diastolic dysfunction.

Subcritical stenosis was detected in the coronary angiography. Risk scorings were calculated for open heart surgery, EUROSCORE II was found $\% 15.6$ and STS was found \%11. Then in cardiology and cardiovascular surgery council, he was accepted as a high risk patient for surgery and TAVR was decided to be performed.

Aortic tomographic angiography was performed to evaluate the suitability of the patient for TAVI. Tomography showed that aortic anulus was suitable for operation with $26 \mathrm{~mm}$ Star Edwards Saphien XT balloon expandable aortic bioprosthetic valve. However, under the renal artery, a dissection flap was observed at a distance of $3 \mathrm{~cm}$ from the iliac bifurcation in the abdominal aorta. At the flap location, the aortic true lumen was measured as $4 \mathrm{~mm}$ in the narrowest area. The lesion length including lesion and solid segment was $36 \mathrm{~mm}$, the lesion' diameter was $13 * 15.5 \mathrm{~mm}$ in the beginning, and the end was $13 * 16 \mathrm{~mm}$ (Figure-1). As the aortic lumen diameter required for transfemoral use of the valve was $6.5 \mathrm{~mm}$, true lumen, in the dissection area was not suitable for the placement .

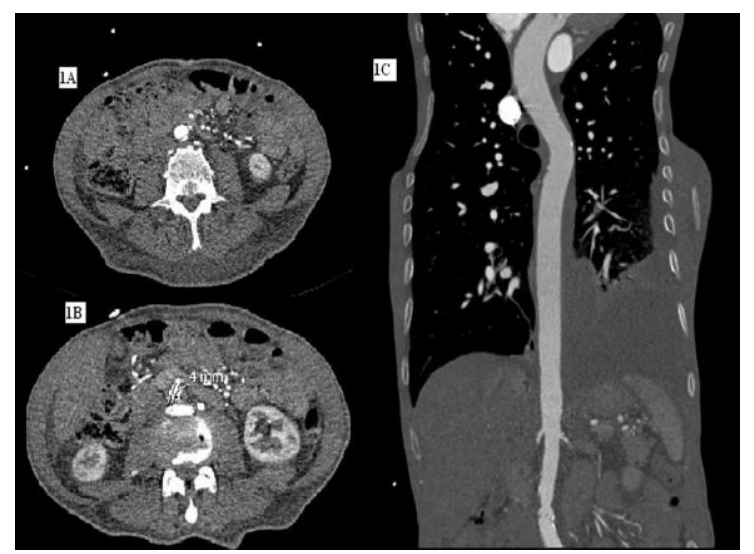

Figure1. Tomographic angiogram of the patient shows the appearance of abdominal aortic dissection and the narrowest luminal diameter at this localization is $4 \mathrm{~mm}$. 1A:Appearance of the dislocation flap is observed. 1B: $4 \mathrm{~mm}$ luminal diameter. 1C: Coronal image shows abdominal aortic dissection.

For this reason, it is planned to continue the TAVI procedure after the dissection and stenosis regions are treated firstly with EVAR method in the same session. After local anesthesia, right femoral artery was punched and 2 proglide vascular closure systems were placed. Right Judgins Cathater (6F) was passed through the dissection and stenosis over therimo guide wire and replaced with an amplatzer super stiff guide wire. Firstly, EndurantII $23 * 23 * 70$ $\mathrm{mm}$ (Medtronic) graft coated self expandable stent was implanted from the renal artery level to the distal aorta, followed by postdilatation with a Reliant AB46 balloon(Medtronic). The images taken after the procedure showed no leak in the graft site and the luminal clearance required for TAVI was provided (Figure-2).

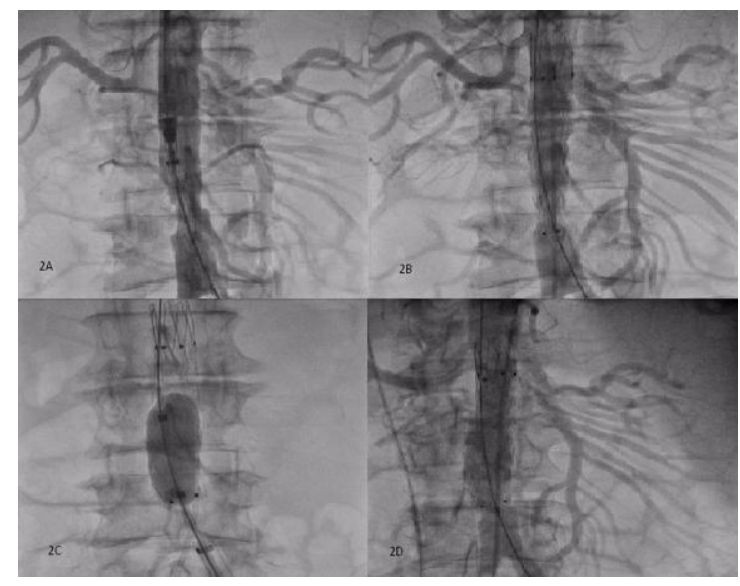

Figure2. EVAR operation is seen. 2A: The system was placed in the lesion area. $2 B$ : The system is switched on and it is seen that there is a residual stenosis after the operation. 2C: Postdilated. 2D: After ballooning it was seen that there was no residual lesion and the procedure was completed successfully.

Then sheath of the valve was placed over the same guide wire. Then aortic valve was passed with $6 \mathrm{~F}$ AL-1 catheter and 0.35 straight guide wire. Straight guide wire replaced with Safari Guide Wire (Boston Scientific). The aortic valve was predilatated with a $23 \mathrm{~mm}$ aortic balloon under rapid pacing. Then $26 \mathrm{~mm}$ STAR EDWARDS SAPHIEN XT (Edwards Lifesciences) balloon expandable aortic bioprosthesis valve was implanted under rapid pacing.

There was complete abolition of trans-aortic gradient and angiography post-TAVI showed no 
aortic regurgitation and complication. (Figure $3)$.

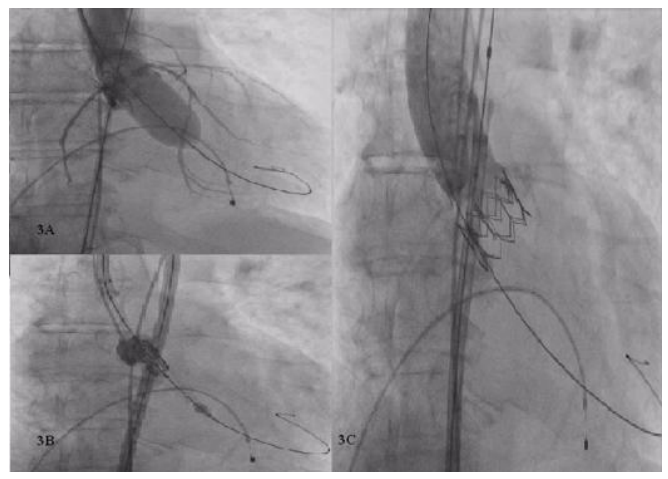

Figure3. TAVI operation is seen. 3A: Predilatation phase is seen. 3B: Bioprosthesis valve placed in aortic valve position. 3C: Image taken after cover implantation.

After the procedure, the patient was transfered to intensive care unit and underwent a hemodialysis session due to opaque nephropathy. The patient subsequently made an excellent recovery and was discharged one week after the operation. With one year follow-up, the patient was well and asymptomatic. He was able to have a very active daily life without limitation. Follow- up echocardiography showed a well-seated aortic valve prosthesis velocity of $1.8 \mathrm{~m} / \mathrm{sn}$ with a peak trans-aortic gradient of 10 $\mathrm{mm} \mathrm{Hg}$ and good left ventricular function (LVEF: \%60). No valvular regurgitation was seen. No evidence of abdominal aortic leak was seen on ultrasonographic follow-up.

\section{DISCUSSION}

Aortic stenosis is increasingly prevalent in the elderly population. A recent study showed that nearly $5 \%$ of 75 -year olds have evidence of significant aortic stenosis . Abdominal aortic pathology occur in approximately $5 \%$ of men over the age of 50 years (2) . Conventional aortic valve surgery in such patients carries a prohibitively high risk, which is further amplified by the presence of abdominal aorta pathology . Similarly, treatment of abdominal aorta pathology in the presence of critical aortic stenosis carries an unacceptably high risk (4).

It is crucial for TAVI to know the individual anatomical details prior to the procedure in order to make satisfactory planning of the procedure and proper prosthesis choice and patient selection. Among the imaging modalities available for the evaluation of patients prior to TAVI, computed tomography (CT) plays a central role in patient selection and planing prior TAVI (1).
Our case supported the opinions that suggest preoperative evaluation of the entire aortic tract as well as aortic annulus by computed tomography. Because there was no clinical evidence to suggest an abdominal aortic dissection in our case but the current dissection was found incidentally on computed tomography prior to TAVI. Unless the dissection and aortic luminal stenosis were noticed, at the beginning of the procedure we could encounter a lethal complication such as aortic rupture as a result of sheath intervention.

TAVI procedure could be performed via alternative interventional sites due to aortic dissection and aortic luminal stenosis that block femoral intervention in our case(5). However, we preferred transfemoral method as it is easier to perform and with better clinical results than other methods. The transfemoral approach has the advantage that procedures can be performed percutaneously and under local anesthesia which is an important modality in elderly patients with co-morbidities and significant anesthetic risks (6).

Because of the possibility of hemodynamic disturbance provoked by aortic stenosis that can occur during EVAR procedure, firstly TAVI and later in another session EVAR precedure could have been done, by using alternative interventional approaches (7). However, due to the negative clinical consequences of hemodynamic changes that may occur after TAVI, we performed the EVAR and TAVI procedures in the same session (8). The consequence of correcting the aortic stenosis in post TAVI patients is increasing risk of the rupture of the aortic dissection due to the increased systolic pressure and strain within the dissection(9) .

\section{CONCLUSION}

Our case highlights the importance of detailed assessment in all patients before consideration of TAVI. Computed tomography has central importance among the imaging modalities available for evaluating patients prior to TAVI. It can reliably acquire all imaging-based parameters required for adequate patient selection and procedure planning in one scan.

The increasing uptake of TAVI in elderly, highrisk patients are likely to uncover an increasing number of patients with both aortic stenosis, aortic dissection and aneurysm which may lead to complications that require treatment. 
The evaluation of patients prior to TAVI in this respect is important for clinical outcomes. The combination of careful assessment, improved trans-catheter techniques can together enable the simultaneous treatment of some complex cardiovascular conditions, previously treated surgically.

\section{REFERENCES}

[1] L. Lehmkuhl, B. Foldyna, M. Haensig, K. von Aspern, et al. Role of Preprocedural Computed Tomography in Transcatheter Aortic Valve Implantation. Fortschr Röntgenstr 2013; 185: 941-949

[2] M.Drury-Smith, A. Garnham, S. Khogali. Critical Aortic Stenosis in a Patient with a Large Saccular Abdominal Aortic Aneurysm: Simultaneous Transcatheter Aortic Valve Implantation and Drive-by Endovascular Aortic Aneurysm Repair. Catheterization and Cardiovascular Interventions 80:1014-1018 (2012)

[3] M.Marwan, S. Achenbach. Role of Cardiac CT Before Transcatheter Aortic Valve Implantation (TAVI). Curr Cardiol Rep (2016) 18: 21 DOI 10.1007/s11886-015-0696-3

[4] Brewster, D, Cronenwett J, Hallett J, Johnston W, Krupski W,Matsumura J. Guidelines for the treatment of abdominal aortic aneurysms, Report of a subcommittee of the Joint
Council of the American Association for Vascular Surgery and Society for Vascular Surgery. J Vasc Surg 2003;37:1106-1117.

[5] Ferrari E, von Segesser LK. Transcatheter aortic valve implantation (TAVI): State of the art techniques and future perspectives. Swiss Med Wkly 2010;140:w13127.

[6] Pascual I, Carro A, Avanzas P, HernándezVaquero D, et all. Vascular approaches for transcatheter aortic valve implantation. J Thorac Dis. 2017 May;9(Suppl 6):S478-S487. doi: 10.21037 /jtd.2017.05.73

[7] M.Drury-Smith , A. Garnham, and Saib Khogali. Sequential Trans-Catheter Aortic Valve Implantation and Abdominal Aortic Aneurysm Repair. Catheterization and Cardiovascular Interventions 79:784-788 (2012)

[8] Gotzmann M, Hehen T, Germing A, Lindstaedt $\mathrm{M}$, et all. Short term effects of transcatheter aortic valve implantation on neurohormonal activation, quality of life and 6 minute walk test in severe and symptomatic aortic stenosis. Heart 2010;96:1102-1106.

[9] Vorp DA, Raghavan ML, Webster MW. Mechanical wall stres in abdominal aortic aneurysm: Influence of diameter and asymmetry. J Vasc Surg 1998;27:632-639.

Citation: Refik Emre Altekin, All Yasar Kllinc, Gorkem Goldag Critical Aortic Stenosis in a Patient with a Abdominal Aort Dissection and Narrowed: Simultaneous Transcatheter Aortic Valve Implantation and Endovascular Aortic Repair. ARC Journal of Clinical Case Reports. 2017; 3(4):10-13. doi:dx.doi.org/ 10.20431/2455-9806.0304003.

Copyright: () 2017 Authors. This is an open-access article distributed under the terms of the Creative Commons Attribution License, which permits unrestricted use, distribution, and reproduction in any medium, provided the original author and source are credited. 